\title{
A STUDY ON SURGICAL MANAGEMENT OF THYROTOXICOSIS
}

\author{
Lakshmi Meena Jasti¹, Obed Ahmed Shaik2, Beenaboina Venkata Ramana Murthy33, Padala Chandra Mohan ${ }^{4}$
}

${ }_{1}^{1}$ Assistant Professor, Department of General Surgery, ACSR Government Medical College, Nellore, Andhra Pradesh, India.

${ }^{2}$ Senior Resident, Department of General Surgery, ACSR Government Medical College, Nellore, Andhra Pradesh, India.

${ }^{3}$ Assistant Professor, Department of General Medicine, ACSR Government Medical College, Nellore, Andhra Pradesh, India.

${ }_{4}^{4}$ Assistant Professor, Department of General Surgery, ACSR Government Medical College, Nellore, Andhra Pradesh, India.

\begin{abstract}
BACKGROUND

Thyrotoxicosis refers to a clinical condition that results from high thyroid hormone action in tissues generally due to high tissue thyroid hormone levels ${ }^{1}$ Hyperthyroidism refers to over activity of the thyroid gland leading to high synthesis and excessive production of thyroid hormones ${ }^{1}$. The underlying problem in patients with thyrotoxicosis is acceleration of many physiological processes, and the clinical manifestations reflect that acceleration. We wanted to study the clinical pattern of presentation of patients with thyrotoxicosis, indications for surgery in thyrotoxicosis patients, functional results, and quality of life following the surgical treatment.
\end{abstract}

\section{METHODS}

This is a prospective study in the surgical department of the ACSR Govt. Medical College and Hospital, from December 2017 to December 2018. Totally thirty patients with varied clinical presentations, confirmed to be suffering from the thyrotoxicosis, and after tests were appropriately evaluated, thyrotoxicosis controlled, consenting patients were operated. Clinical details were entered onto a proforma specification. Data was entered in a Microsoft excel, computers read sheet and analysed by using WHO epidemiological information package.

\section{RESULTS}

Surgical management of hyperthyroidism provides effective endocrinal control if surgery is complete. Patients should be fully informed of all possible postoperative complications that could occur, especially vocal cards. Long-term follow-up is necessary to detect recurrence, which can occur more than 20 years after partial thyroidectomy surgery. Total thyroidectomy is reserved for patients with severe disease or very large goiters in which recurrences will be highly problematic, but has an increased risk of hyperparathyroidism and laryngeal nerve damage.

\section{CONCLUSIONS}

Thyrotoxicosis occurs due to rise in thyroid hormone synthesis, its secretion by the thyroid gland, and its consequences on various systems with altered biochemical levels. This most commonly manifests as Diffuse Toxic Goiter and Toxic Multinodular Goiter. The management of thyrotoxicosis is mainly on three treatment modalities, anti-thyroid medication, radioactive iodine ablation or surgery. Surgery plays an important role in the management of thyrotoxicosis with immediate and cost-effective treatment with minimal morbidity and mortality. Total thyroidectomy is the surgery of choice in Graves' disease, while a total thyroidectomy or thyroid lobectomy are performed in patients with toxic nodular goiters. Although thyroid surgery can be associated with significant complications, in many operative centers, surgery provides effective long-time resolution of hyperthyroidism and hence should be considered an integral part of treatment rather than the last choice for clinicians. Surgery should be considered as an immediate and completely effective solution for thyrotoxicosis, especially when compared with long duration medical therapy, because it can provide an observable improvement in the quality of life (QOL) of the patients.

HOW TO CITE THIS ARTICLE: Jasti LM, Shaik OA, Murthy BVR, et al. A study on surgical management of thyrotoxicosis. J. Evolution Med. Dent. Sci. 2019;8(21):1708-1712, DOI: 10.14260/jemds/2019/376

\section{BACKGROUND}

Kocher encountered the striking malady of exophthalmic goiter, as describe by Grave's ${ }^{2}$ preoperatively. Whereas his contemporaries would stay away from such risky thyrotoxic patients, Kocher designed staged surgery for them. He was doing a first stage of ligation of several named thyroid arteries to diminish the gland's blood supply, then a second stage of one-sided lobectomy.

'Financial or Other Competing Interest': None.

Submission 04-04-2019, Peer Review 11-05-2019,

Acceptance 17-05-2019, Published 27-05-2019.

Corresponding Author:

Dr. Obed Ahmed Shaik,

S/o. Shaik Sagir Ahmed, 24-6-28, Pride Gardens,

Saraswathi Nagar, Magunta Layout,

Nellore-524003, Andhra Pradesh, India.

E-mail: obedshaikat1@gmail.com

DOI: $10.14260 /$ jemds $/ 2019 / 376$
If thyrotoxicosis remained or recurred, then a third stage was considered to reduce the size of the remaining lobe. Kocher's mortality rate for such cases was only $4.5 \%$, which was extraordinary, especially when reviewing that this was a time before preoperative preparation with iodine or antithyroid drugs. ${ }^{3}$

Charles mayo was dramatically introduced to thyroid surgery in 1890, and he moved to tackle feared problem of hyperthyroidism. Mayo had performed 278 successful surgeries for Graves's disease with mortality less than $1 \% .4$ with highest no. of surgeries and lowest mortality, hence was titled "father of American thyroid surgery".

The most notable thyroid surgeons were Emil Theodor Kocher (1841-1917) is regarded by many as Father OF Thyroid Surgery, \& Theodor Billroth (1829 - 1894).

In 1909, Kocher Received Nobel Prize for medicine in recognition for his works on physiology, pathology \& surgery of thyroid gland. 5 
As we know toxic nodular goiter is less common than Graves' disease, its prevalence increases with age and in the presence of iodine deficiency.

Hence, toxic nodular goiter can be actually more common than Graves' disease in older patients from regions of iodine deficiency. ${ }^{6}$

\section{Aims and Objectives}

To study the Clinical pattern of presentation of patients with thyrotoxicosis, Indication for surgery in thyrotoxicosis patients and to assess the functional results and quality of life following the surgical treatment

\section{METHODS}

This is a prospective interventional study in the surgical department of the ACSR Govt. Medical College and Hospital from December 2017 to December 2018. Got approval from IEC, consent was taken from all patients

Totally thirty patients with varied clinical presentations are confirmed to be suffering from the thyrotoxicosis and tests were appropriately evaluated and Thyrotoxicosis controlled, and consented patients were operated.

This study was conducted in association with department of General Medicine.

\section{Inclusion Criteria}

1. Patients admitted with signs and symptoms of thyrotoxicosis and with confirmed elevated thyroid hormone levels.

2. Patients with clinical features of thyrotoxicosis with or without treatment.

\section{Sample Size}

A total of thirty patients were included in this study

$$
\left[\mathrm{n}=4 \mathrm{pq} / \mathrm{d}^{2}\right] \mathrm{p}=50, \mathrm{q}=50, \mathrm{~d}=10
$$

is the formula used to calculate sample size, where $n=$ sample size, $\mathrm{p}=$ prevalence in $\%, \mathrm{q}=100-\mathrm{p}, \mathrm{d}=$ precision.

\section{Pre-Operative Assessment}

All the patients were subjected to basic investigations like complete hemogram, blood sugar, urea, urine analysis, chest $\mathrm{x}$-ray, ultrasound and other specific investigations like. Indirect laryngoscopy, serum calcium, cervical x-ray and CT scan for suspicious of intra-thoracic extension. Indirect laryngoscopy done for all patients as routine work up. Serum calcium was assessed pre-operatively and were in normal limits. Normal range $8.10-10.40 \mathrm{mg} / \mathrm{dl}$.

This is to have base line value against which it can be compared in post-operative period for diagnosing cases of hypocalcaemia. Cervical x-ray was done for all patients. $\mathrm{Ct}$ scan for suspicious of intrathoracic extension. TSH, T3, T4 levels were estimated. All patients were found to have raised free T3, T4 levels and low TSH levels and have clinical and lab features of thyrotoxicosis, formed the subject of the study.

After the preoperative assessment was done patients were posted for total thyroidectomy. Surgery was done for all cases under general anaesthesia with intubation.

\section{Indications for Surgery}

Large goiter with or without symptoms of compression.
- Failure of ongoing medical treatment.

- Recurrence after anti-thyroid medication.

- $\quad$ Preference for surgery.

All patients controlled with anti-thyroid medication and those patients taken up for surgery if antithyroid drugs was ineffective for primary thyrotoxicosis or After controlling thyrotoxicosis symptoms with medical therapy in case of toxic multinodular goiter; those with large goiter with or without compressive symptoms.

\section{Post-Operative Care}

Drainage and dressing were inspected for soakage on the night of surgery and tube was removed 24-48 hours after surgery if there is no active bleeding. Serum calcium levels post-operatively were monitored $12 \mathrm{hrs}, 24 \mathrm{hrs}$ with preoperative values. If found to be less than $8.0 \mathrm{mg} / \mathrm{dl}$ were started on oral calcium if asymptomatic and oral or intravenous calcium supplementations if symptomatic. Postoperatively thyroid hormones levels were monitored, and thyroxine supplementation was started by $1.7 \mathrm{mcg} / \mathrm{kg}$ body weight.

All 30 patients were in hyperthyroidism state when the surgical option was planned, and they were undergone total thyroidectomy in a state of clinical or biological euthyroidism after medical therapy like antithyroid medications (Methimazole) and also received propranolol for control of cardiac associated thyrotoxic symptoms.

\section{Statistical Analysis}

Data were entered in a Microsoft excel computers read sheet and analysed by using WHO epidemiological information package
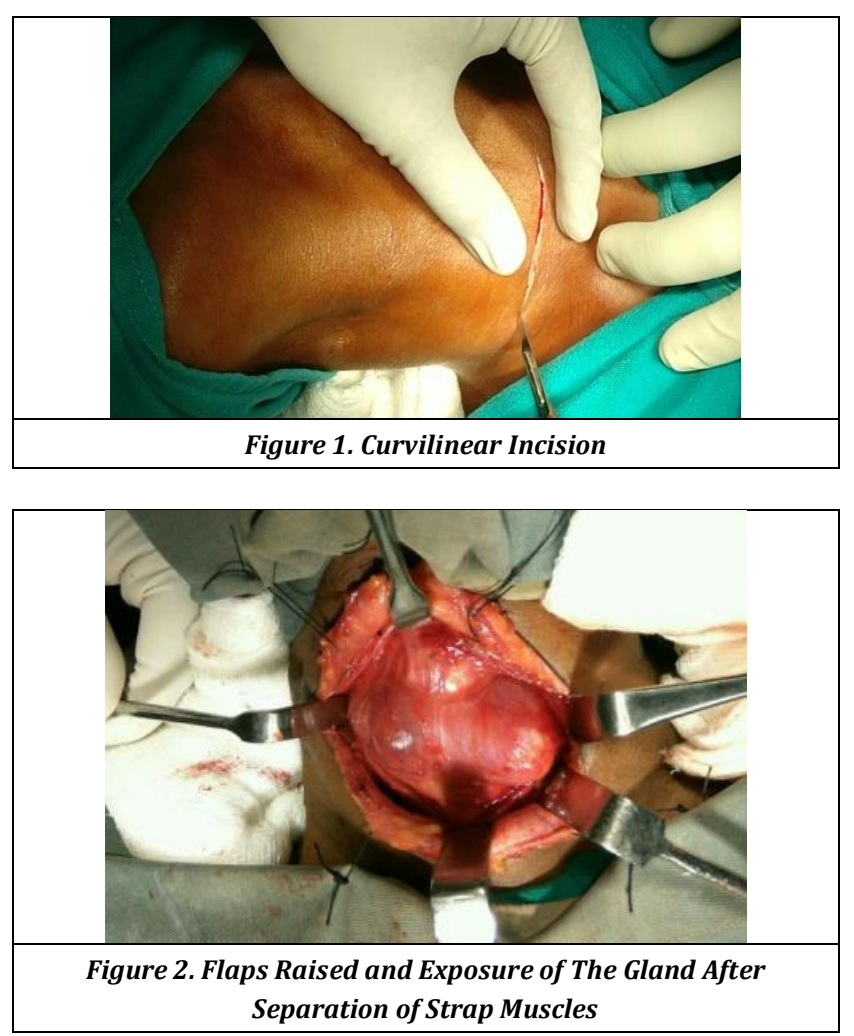

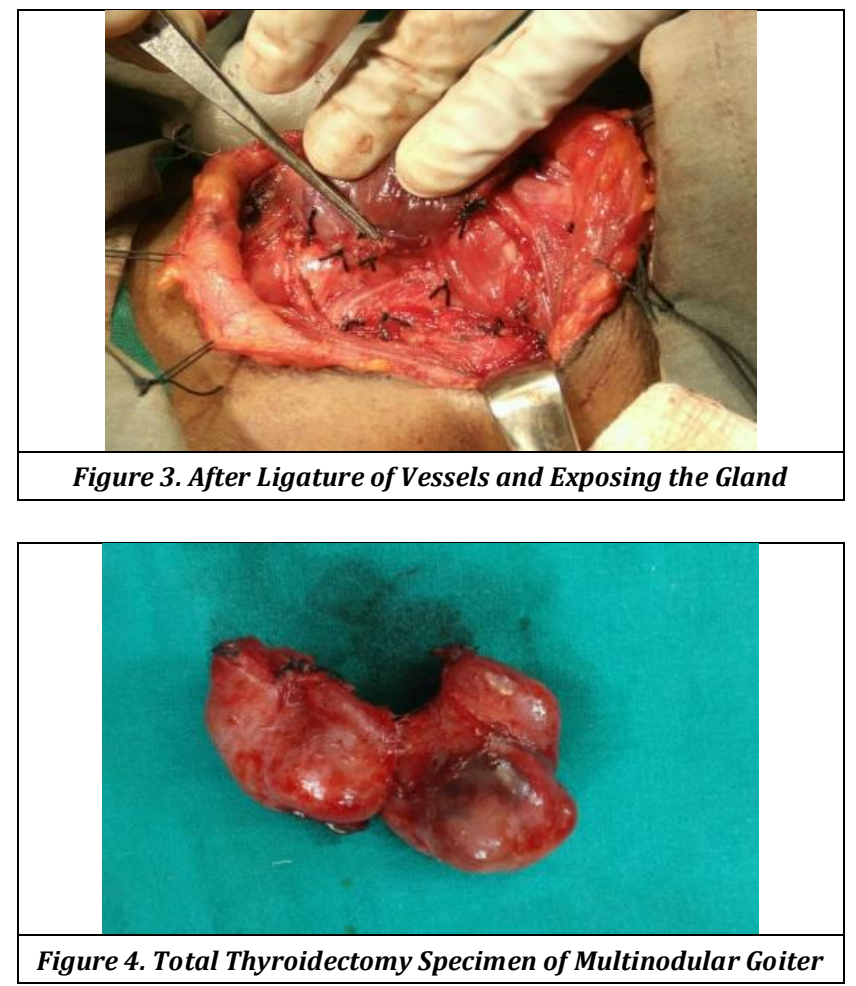

\section{RESULTS}

Surgical management of hyperthyroidism provides effective endocrinal control if surgery is complete. Patients need to be clearly informed of all possible postoperative complications that could occur, especially vocal cards. Long-term follow-up is necessary to detect recurrence, which can occur more than 20 years after partial thyroidectomy surgery.

All the cases studied had goiter. The Incidence of various thyrotoxic signs and symptoms in our study are tabulated below:

\begin{tabular}{|c|c|c|c|}
\hline Sl. No. & Clinical features & $\begin{array}{c}\text { No. of Cases } \\
\text { (N=30) }\end{array}$ & Percentage (\%) \\
\hline 1 & Goitre & ALL & $100 \%$ \\
\hline 2 & Palpitations & 24 & $80 \%$ \\
\hline 3 & Tremors & 16 & $53.33 \%$ \\
\hline 4 & Weight Loss & 9 & $30 \%$ \\
\hline 5 & Heat Intolerance & 5 & $16.67 \%$ \\
\hline 6 & Nervousness & 5 & $16.67 \%$ \\
\hline 7 & Tachycardia & 10 & $33.33 \%$ \\
\hline 8 & Menstrual Abnormalities & 3 & $10 \%$ \\
\hline 9 & Bruit & 2 & $6.67 \%$ \\
\hline 10 & Eye Signs & 3 & $10 \%$ \\
\hline \multicolumn{3}{|c|}{ Table 1. Frequency of Thyrotoxic Symptoms (N=30) } \\
\hline
\end{tabular}

\begin{tabular}{|c|c|c|c|}
\hline \multicolumn{2}{|r|}{ Indications } & $\begin{array}{l}\text { No of Cases } \\
(\mathrm{N}=30)\end{array}$ & Percentage \\
\hline \multirow{2}{*}{$\begin{array}{l}\text { Large } \\
\text { goiter }\end{array}$} & $\begin{array}{c}\text { Without compressive } \\
\text { symptoms }\end{array}$ & 5 & $16.67 \%$ \\
\hline & $\begin{array}{c}\text { With compressive } \\
\text { symptoms }\end{array}$ & 3 & $10 \%$ \\
\hline \multicolumn{2}{|r|}{ Failure of treatment } & 15 & $50 \%$ \\
\hline \multicolumn{2}{|r|}{ Recurrence after therapy } & 2 & $6.6 \%$ \\
\hline & Preference for surgery & 5 & $16.67 \%$ \\
\hline \multicolumn{4}{|c|}{ Table 2. Indications for Surgery $(\mathrm{N}=30)$} \\
\hline
\end{tabular}

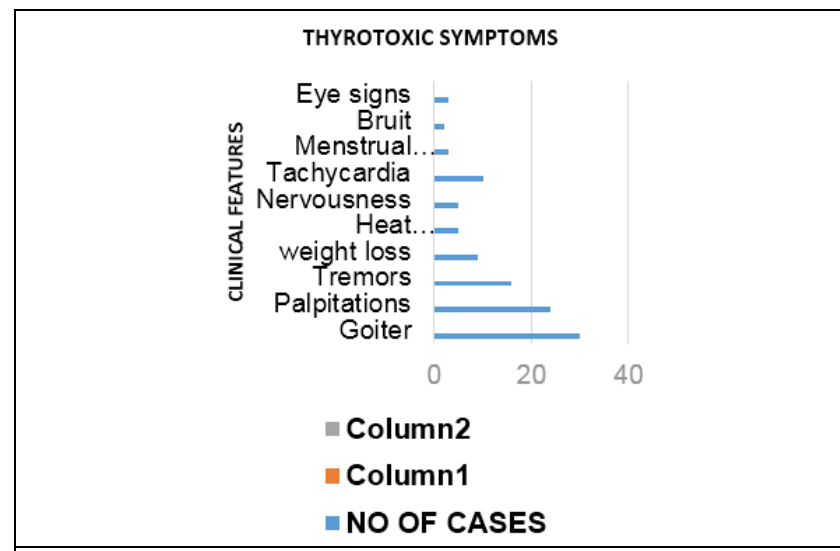

Graph 1. Graphical Presentation of Thyrotoxic Symptoms $(N=30)$ Most common presenting feature is goiter and next most common patients presented this study is palpitations.

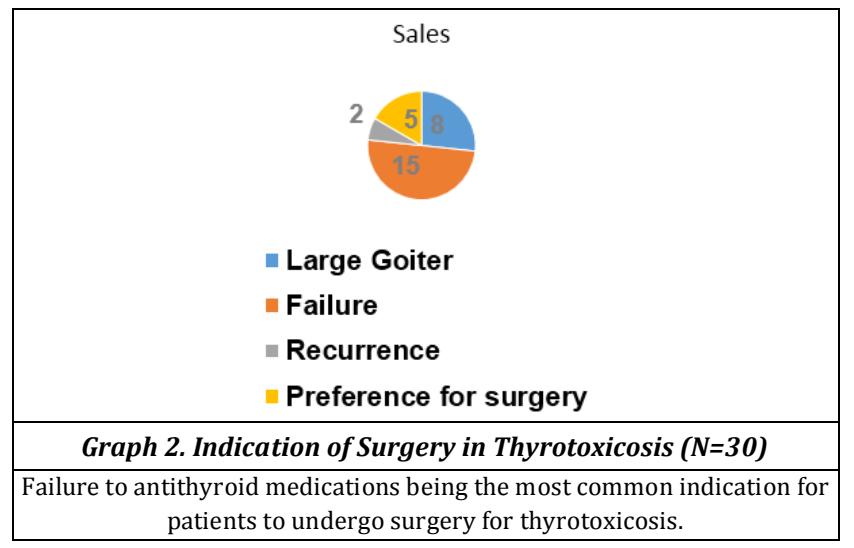

\begin{tabular}{|c|c|c|}
\hline \multicolumn{3}{|c|}{ Quality of Life } \\
\hline POD & No. of Cases & Percentage \\
\hline $7^{\text {th }}$ & 4 & $13.3 \%$ \\
\hline $9^{\text {th }}$ & 5 & $16.67 \%$ \\
\hline $10^{\text {th }}$ & 9 & $30 \%$ \\
\hline $1^{\text {th }}$ & 12 & $46.6 \%$ \\
\hline \multicolumn{2}{|c|}{ Table 3. Ability to Attend Work and Return of Daily Activities } \\
(N=30) 2
\end{tabular}

\section{DISCUSSION}

The clinical syndrome of thyrotoxicosis can be caused by many diseases, the two most common causes are Graves' Disease (GD) and Toxic Nodular Goiter (TMNG).

In this study, toxic nodular goiter constituted almost 73\% of the cases. Most of the patients who were diagnosed to have thyrotoxicosis were in the age group of 40 - 50 years.

In our study all the patients had goiter. The most significant clinical features were palpitation, tremors, weight loss, heat intolerance, excitability, menstrual abnormality \& bruit are in the order of frequency.

TSH, Total T4, Total T3 was done for all the cases. In all the cases the TSH was reduced \& $\mathrm{T} 4$ \& $\mathrm{T} 3$ was raised confirming the hyperthyroid state \&indicating the severity of the disease.

All the patients in whom surgery was planned, were given antithyroid drugs\& taken up for surgery after adequate control of toxicity. This prevented the complication of thyroid storm in the preoperative $\&$ in the post-operative period. 
After giving antithyroid medications, euthyroid state was attained between 2 weeks to maximum of 8 weeks.

\section{Present Study}

The main goal of this study was to know the surgical indications and the effective outcome of total thyroidectomy in the treatment of thyrotoxicosis and quality of life after surgery.

In total 30 patients the indications for total thyroidectomy were: 8 large goiters with compressive symptoms, 15 patients with failure or intolerant to previous treatment, 2 patients with recurrent hyperthyroidism after medical treatment; 5 patients with preference for surgery.

The average postoperative hospital stay was 7 days (Range: 5-9). Transient hypocalcaemia occurred in 2 patients $(6.6 \%)$ and transient unilateral recurrent laryngeal nerve injury in other 2 patients (3.3\%). None of the patients had neither permanent hypocalcaemia nor permanent recurrent laryngeal nerve injury.

All 30 treated patients got relieved of their symptoms and became biochemically hypothyroid after the operation. All patients after Total thyroidectomy medication were kept on single medication on thyroxine only except 2 patients additionally were on calcium supplements for hypocalcaemia.

Thus, after total thyroidectomy results were fast, reliable decrease of hyperthyroidism and removal of multinodular goitre, needs no re-treatment, removes any coexisting malignancy, and post-surgical hypothyroidism is simple to treat.

Total thyroidectomy is reserved for patients with severe disease or large goiters in whom recurrences would be highly problematic but has an increased risk of hyperparathyroidism and laryngeal nerve damage.

By AM Fam physicians of American family physicians of hyperthyroidism stated that Risk of hypothyroidism (25 Percent) or hyperthyroid relapse (8 Percent); temporary or permanent hypoparathyroidism or laryngeal paralysis (Less than 1 Percent); higher morbidity and cost than radioactive iodine; requires patient to be euthyroid preoperatively with antithyroid drugs or iodides to avoid thyrotoxic crisis. ${ }^{7}$

Ahmed Al-Adhami, et al.., Of 71 AF procedures: one developed acute airway obstruction; one permanent RLN palsy; four permanent hypocalcaemia; and none developed recurrent toxicity. There were nil deaths within a year of surgery and ablative surgery eliminated the need for lifelong specialist follow-up, with an insignificant rise in permanent hypocalcaemia. ${ }^{8}$

Postoperative complications like four\% permanent recurrent laryngeal nerve palsy, nine $\%$ hematoma requiring surgical revision, and three\% definitive hypocalcaemia. Postoperative complications were more frequent in multinodular goiter than in Graves' disease.

Pisanu A et al.., surgical indications and the effectiveness of total thyroidectomy in the treatment of 70 toxic MNG. All 70 treated patients relieved their symptoms and became biochemically hypothyroid after the operation. Total thyroidectomy results in a rapid, reliable resolution of hyperthyroidism and removal of multinodular goitre, requires no re-treatment.
Branka Bukvic, MD et al.., The QoL of the GD patients was worse than that of the TNG patients, with significant differences in eye symptoms, anxiety, and sex life, preoperatively, and in eye symptoms, anxiety, emotional susceptibility, and overall QoL postoperatively.

The improvement in QoL in the GD patients was significant after surgical treatment. In the TNG patients, the improvement was significant in all but one. The QoL of GD patients is worse than those of TNG patients. Surgery may improve QoL in patients with GD and TNG even if they have reached required thyroid status with medication treatment, preoperatively.

\section{CONCLUSIONS}

Thyrotoxicosis occurs due to rise in thyroid hormone synthesis and its secretion by the thyroid gland and its consequences on various systems with altered biochemical levels. This most commonly manifests as Diffuse Toxic Goiter and Toxic Multinodular Goiter. The management of thyrotoxicosis is mainly on three treatment modalities, like anti-thyroid medication, radioactive iodine ablation or surgery.

Surgery plays an important role in the management of thyrotoxicosis with immediate and cost-effective treatment with minimal morbidity and mortality. Total thyroidectomy is the surgery of choice in Graves' disease, while a total thyroidectomy or thyroid lobectomy are performed in patients with toxic nodular goiters.

Although thyroid surgery can be associated with significant complications, in many operative centers surgery provides effective long-term resolution of hyperthyroidism and hence should be considered an integral part of treatment rather than the last choice for clinicians.

Surgery should be planned as an immediate and totally effective solution for thyrotoxicosis, especially when compared with prolonged medical therapy, because it can provide a demonstrable improvement in the quality of life (QOL) of the patients.

\section{REFERENCES}

[1] Bahn RS, Burch HB, Cooper DS, et al. Hyperthyroidism and other causes of thyrotoxicosis: management guidelines of the American Thyroid Association and American Association of Clinical Endocrinologists. Endocr Pract 2011;17(3):456-520.

[2] Graves RJ. Newly observed affection of the thyroid gland in females. Clin Lect Lond Med Surg J 1835;7:516-7.

[3] Hannan SA. The magnificent seven: a history of modern thyroid surgery. International Journal of Surgery 2006;4(3):187-91.

[4] Mayo CH. Surgery of the thyroid: observations on 5000 Operations. JAMA 1913;61(1):10-3.

[5] Becker WF. Presidential address: Pioneers in thyroid surgery. Ann Surg 1977;185(5):493-504.

[6] Laurberg P, Pedersen KM, Vestergaard H, et al. High incidence of multinodular toxic goitre in the elderly population in a low iodine intake area vs. high incidence of Graves' disease in the young in a high iodine intake area: comparative surveys of 
thyrotoxicosis epidemiology in East-Jutland Denmark and Iceland. J Intern Med 1991;229(5):415-20.

[7] Reid JR, Wheeler SF. Hyperthyroidism: diagnosis and treatment. American Family Physicians 2005;72 (4):623-30.

[8] Al-Adhami A, Snaith AC, Craig WL, et al. Changing trends in surgery for Graves' disease: a cohort comparison of those having surgery intended to preserve thyroid function with those having ablative surgery. Journal of Otolaryngology - Head \& Neck Surgery 2013;42(1):37.

https://doi.org/10.1186/1916-0216-42-37 